\title{
Sleepiness in Various Shift Combinations of Irregular Shift Systems
}

\author{
Mikael SALLINEN ${ }^{1 *}$, Mikko HÄRMÄ̈${ }^{1}$, Pertti MUTANEN², \\ Riikka RANTA ${ }^{2}$, Jussi VIRKKALA ${ }^{1}$ and Kiti MÜLLER ${ }^{1}$
}

\author{
${ }^{1}$ Brain and Work Research Units, Finnish Institute of Occupational Health, Topeliuksenkatu 41 a A, 00250 Helsinki, \\ Finland \\ ${ }^{2}$ Department of Epidemiology and Biostatistics, Finnish Institute of Occupational Health
}

Received October 15, 2004 and accepted November 16, 2004

\begin{abstract}
The present study examined the occurrence of sleepiness in various shift combinations ending with a night or morning shift. Three weeks' sleep/work shift diary data, collected from 126 randomly selected train drivers and 104 traffic controllers, were used in statistical analyses. The occurrence of sleepiness at work (i.e., Karolinska Sleepiness Scale 7 or higher) was tested with a generalised linear model with repeated measurements including explanatory factors related to shifts, sleep, and individual characteristics. The prevalence of severe sleepiness varied between $25 \%$ and $62 \%$ in the combinations ending with a night shift and between $12 \%$ and $27 \%$ in the combinations ending with a morning shift. The occurrence of sleepiness did not, however, systematically vary between the shift combinations in either case. An increased risk for sleepiness was associated with high sleep need and long shift duration in the night shift and with high sleep need, short main sleep period, long shift duration and an early shift starting time in the morning shift. Also having a child was associated with an increased risk for sleepiness in the night shift. The results suggest that the shift history of 24-36 h prior to the night and the morning shift is not strongly associated with the occurrence of sleepiness at work, but there are other factors, such as shift length and starting time and sleep need, that affect a risk for sleepiness at work.
\end{abstract}

Keywords: Shift work, Sleep, Sleepiness, Train drivers, Railway Traffic controllers

\section{Introduction}

Insufficient sleep and impaired alertness are the most common problems with non-physiological working hours $^{1,2)}$. In night shifts, both main determinants of alertness, time since sleep and the circadian phase have a disadvantageous effect on the maintenance of alertness. In the early morning shift, the preceding main sleep period is usually reduced by about $2 \mathrm{~h}$ due to the difficulty to fall asleep early in the evening. In addition, one has to rise from bed around the circadian nadir of alertness.

Insufficient sleep and sleepiness are particularly problems in occupations, in which a drop in vigilance can compromise

*To whom correspondence should be addressed. safety ${ }^{3)}$. Thus, sleep and sleepiness at work have been studied especially in industrial and transportation occupations ${ }^{4-8}$. Our recent study on sleepiness in train drivers and railway traffic controllers showed that an increase in the occurrence of severe sleepiness at work is associated with night and morning shifts, long duration of a shift, young age, and a short main sleep period before the shift ${ }^{9)}$. The time-off period before the shift was not consistently associated with the occurrence of severe sleepiness. Interestingly, the time-off period of $16 \mathrm{~h}$ or more before the night shift was associated with a somewhat higher risk for severe sleepiness than the time-off period of 8-16 h. For the morning shift, the situation was the opposite: a short free time period prior to the shift (less than $8 \mathrm{~h}$ ) was associated with an increased risk for severe sleepiness in comparison to a long free time period 
( $16 \mathrm{~h}$ or more). However, the occurrence of severe sleepiness in specific shift combinations ending with either the night or morning shift were not examined. In addition, the significance of daytime naps prior to the night shift was not studied.

Our next study examined the sleep-wake rhythm in specific shift combinations ending with either the night or morning shift ${ }^{10)}$. The main findings were that the main sleep period curtailed particularly in Evening+Morning and Morning+Night shift combinations, in addition to two successive night shifts. On the other hand, afternoon napping prior to the night shift increased in the Morning+Night combination. The increased daytime napping in this shift combination was probably due to an early wake-up time and also to good possibilities to nap in the midway of a round trip. Also the starting time of the shift played a significant role. On average, a delay of one hour in the starting time of the night shift increased the chance for taking an afternoon nap by half. One hour delay in the starting time of the morning shift, in turn, increased the main sleep period by $44 \mathrm{~min}$. We also explored dozing off at work, but at least all the train drivers, who were interviewed afterwards, said that all dozing offs occurred when the train was stopped. Thus, we could not directly estimate the occurrence of severe sleepiness in the morning and night shifts that were preceded by a different shift history.

The new viewpoint of the present study was to examine the occurrence of severe sleepiness in various shift combinations ending with a morning or night shift in train drives and traffic controllers. In addition, we were interested to study how other shift-related factors (e.g., shift starting time), prior sleep and individual factors (e.g., experienced sleep need) affected the occurrence of severe sleepiness specifically in the morning and night shifts.

\section{Material and Methods}

\section{Subjects}

Of all professional train drivers (drivers, $\mathrm{n}=1,277$ ) from East and South Finland and traffic controllers (controllers, $\mathrm{n}=453$ ) from South, East and West Finland, 230 drivers and 270 controllers were allotted to the study. Of all the allotted drivers and controllers 139 (60.4\%) and 138 (51.1\%), respectively, gave an informed consent to participate in the study. The drives worked in both local (12\%) and longdistance traffic (42\%). The work of the rest of the drivers included both local and long-distance traffic, service driving, duties at a railway depot or a railway yard, as well as, office work. Unfortunately, we did not systematically ask the type of the work task in each shift and thus could not control for this factor.

A total of 47 subjects were removed prior to statistical analyses. Two subjects were excluded because of acute sickness absence and twelve subjects because they were not working on an irregular 3-shift work during the data collection period. The data of eleven subjects were too incomplete for analyses for various reasons (on-call shifts, sick leaves, training courses). In addition, the data of 26 women were excluded because the number of women was too small to examine the gender effect and secondly, all of them were traffic controllers, which would have made it difficult to compare the occupational groups. After these partially overlapping exclusionary criteria, 126 drivers and 104 controllers were included in the statistical analyses. The occupational groups were comparable in terms of age (drivers: mean $42.3 \mathrm{yr}$, range $33-55 \mathrm{yr}$, controllers: mean $44.2 \mathrm{yr}$, range 26-61 yr), shift work experience (drivers: mean $22 \mathrm{yr}$, range 14-36 yr, controllers: mean $19 \mathrm{yr}$, range $<1 \mathrm{yr}-38 \mathrm{yr}$ ), and percentage of subjects who had children in the same household (drivers: $32 \%$, controllers: $18 \%$ ).

\section{Study protocol}

Each subject visited our sleep laboratory twice. During the first visit, the subjects answered a questionnaire concerning shift work and sleep and a nurse instructed them on how to use a sleep diary. The occurrence of severe sleepiness at work was determined with the nine-point Karolinska Sleepiness Scale (KSS) ${ }^{11}$. The subjects gave their KSS ratings in the beginning and at the end of each shift and at any time during the shift when they felt sleepy (a KSS rating of 6 or higher). When a subject rated 7 or greater on the KSS at least once during the shift, the occurrence of severe sleepiness at work was identified. A KSS rating of 7 ("sleepy but not fighting sleep") has been shown to be accompanied by physiological signs of sleepiness, such as occasional slow eye movements and elevated alpha and theta activity in the $\mathrm{EEG}^{11,12)}$. Individual sleep need was measured with the following question: "How many hours of sleep do you need per day (if you could sleep as long as you like)? In other words, how much sleep do you need to be alert and in good shape at work the next day?"

The subjects started keeping the diary on the day of the first laboratory visit and continued with it for three weeks. They were instructed to keep the diary with them all the time and to record the starting and ending of all shifts, sleep periods, dozing offs and experienced sleepiness. The total amount of coffee, alcohol and drugs used within the past 24 
$\mathrm{h}$ was marked in the sleep diary at bedtime. The subjects were also asked to report sleep latency, number of awakenings and sleep quality upon awakening.

When either a morning or a night shift was preceded by at least two sleep periods, which ended after 10:00 p.m., the main sleep period was the longest of these sleep periods. In 44 cases the duration of the main sleep period could not be defined due to missing data (35 cases) or very fragmented sleep-wake rhythm containing no main sleep period ( 9 cases).

\section{Definitions of the shifts}

The shifts were defined according to the following criteria: night shift—at least $3 \mathrm{~h}$ of the shift, or the whole shift, is between 11:00 p.m. and 6:00 a.m.; evening shift—ends between 7:00 p.m. and 1:59 a.m. and is not a night shift; morning shift—commences between 3:01 a.m. and 6:59 a.m. and is not a night or an evening shift; day shift—-starts between 7:00 a.m. and 1:59 p.m. and is not an evening or a night shift.

\section{Shift combinations}

A total of four shift combinations ending with the night shift contained enough cases for separate shift combination groups (Fig. 1). To make the shift combinations equal in terms of preceding sleep, two criteria were applied. If there was a shift within $24-42 \mathrm{~h}$ before the night shift, it had to start after 6:59 a.m. and end before 9:31 p.m. These criteria confirmed that night sleep before the night shift was not affected by insufficient sleep resulting from an early shift or by a delayed bedtime resulting from a late shift. After the application of these criteria, the identified shift combinations encompassed $71 \%$ of all the cases. The rest of the combinations formed their own category called undefined combinations. For the morning shifts, four separate shift combination groups could be formed (Fig. 2). These cases encompassed $89 \%$ of all the observations.

\section{Statistical methods}

The associations between the work-, sleep-, and individualrelated factors and the occurrence of severe sleepiness at work were tested with one type of generalised linear model for repeated measurements called Generalized Estimating Equations (GEE) model ${ }^{13,14)}$. It can be considered as a logistic regression model for repeated measurements data, although it uses the generalised estimation equations method instead of the maximum likelihood method. The model allows the use of unbalanced data and also subjects with missing data can be included, while in the classical multivariate analysis of repeated measurements, all subjects with missing data are simply deleted. The correlation structure of the repeated measures was compound symmetry, i.e., the correlation between two measurements from the same individual was the same regardless of the time length between them.

Prior to the statistical analyses we studied associations between potentially relevant explanatory variables. Experience in shift work was excluded from the model because it correlated strongly with age (Spearman correlation coefficient $=.63, \mathrm{p}<.001$ in the night shift combinations and $.67, \mathrm{p}<.001$ in the morning shift combinations). All models studying the occurrence of severe sleepiness in the night shift contained the following work-, sleep- and individualrelated explanatory variables: occupation (driver, controller), shift combination type, the length of the shift, the length of the preceding main sleep period, the occurrence of afternoon nap before the shift, age $(<$ or $>=45 \mathrm{yr}$ ), children in the same household (at least one child $<7 \mathrm{yr}$, at least one child between 7-17 yr but no younger ones or no children) and self-reported sleep need. In the case of the morning shift, the explanatory variables were the same, except that the occurrence of afternoon nap was removed from the model and the starting time of the shift was added to the model. The reason for including shift starting time in the model was that the starting time of the morning shift is known to affect the length of the preceding sleep period ${ }^{10)}$.

All the statistical analyses were carried out using the Statistical Analysis System, SAS Version 8 (Sas Institute Inc. 1999). The generalised linear models for repeated measurements were carried out by genmod procedure.

\section{Results}

Shift combinations ending with the night shift or the morning shift

As the results on the prevalence of the various shift combinations and the sleep-wake rhythm in these combinations have been reported in our recent study ${ }^{10)}$, they are not repeated here in detail. For the drivers, the most prevalent shift combination ending with the night shift was the Free I Night shift, but also the Morning+Night shift combination occurred frequently (Fig. 1). Among the controllers, the most prevalent combinations were the Day+Night shift and the Morning+Night shift.

Of the shift combinations ending with the morning shift, the Free I Morning and the Evening I Morning were the most common among the drives. The same held for the Free I Morning and the Evening I Morning combinations among the controllers. 


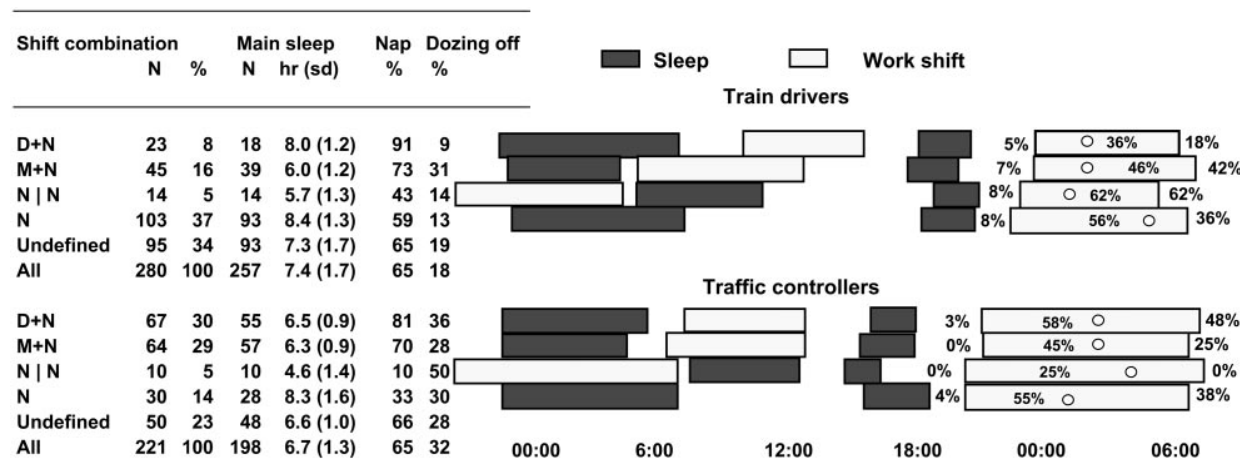

Fig. 1. The prevalence of severe sleepiness (KSS 7 or higher) of the train drivers and traffic controllers in the beginning, at any time (in the middle of the bar) and at the end of the night shift in various shift combinations.

The mean timing of sleep and work periods in the shift combinations are also presented. White dots inside the night shifts denote the mean timing of dozing off at work.

Table 1. A generalised linear model for repeated measurements for the occurrence of severe sleepiness during the night shift

\begin{tabular}{|c|c|c|c|c|c|}
\hline Explaining variable & Categories / units & Odds ratio & $95 \%$ confidence & limits & $\mathrm{p}$ value \\
\hline \multirow[t]{2}{*}{ Age } & $>=45 \mathrm{yr}$ & 1.35 & 0.74 & 2.47 & .32 \\
\hline & $<45$ yr (reference) & 1 & - & - & \\
\hline \multirow[t]{2}{*}{ Occupation } & Driver & 0.94 & 0.55 & 1.60 & .83 \\
\hline & Controller (reference) & & & & \\
\hline \multirow[t]{3}{*}{ Children at home } & $<7 \mathrm{yr}$ & 2.07 & 0.99 & 4.22 & .052 \\
\hline & $7-17 \mathrm{yr}$ & 0.99 & 0.55 & 1.77 & .97 \\
\hline & No children (reference) & 1 & - & - & - \\
\hline Sleep need & Hour & 1.65 & 1.30 & 2.10 & $<.001$ \\
\hline Main sleep period & Hour & 0.92 & 0.77 & 1.09 & .32 \\
\hline \multirow[t]{2}{*}{ Afternoon napping } & yes & 0.68 & .47 & 1.19 & .27 \\
\hline & no (reference) & 1 & - & - & \\
\hline \multirow[t]{5}{*}{ Shift combination } & Undefined & 0.90 & 0.50 & 1.64 & .74 \\
\hline & $\mathrm{D}+\mathrm{N}$ & 0.76 & 0.37 & 1.57 & .45 \\
\hline & $\mathrm{M}+\mathrm{N}$ & 0.71 & 0.35 & 1.44 & .34 \\
\hline & $\mathrm{N}$ IN & 0.63 & 0.20 & 1.98 & .43 \\
\hline & $\mathrm{N}$ (reference) & 1 & - & - & - \\
\hline Shift length & Time in hours & 1.09 & 0.99 & 1.21 & .08 \\
\hline
\end{tabular}

All explanatory variables are included in the model when the effect of one explanatory variable on the occurrence of severe sleepiness at work is tested. A total of 428 observations have been included in the analysis. Driver $=$ train driver, controller $=$ traffic controller, $\mathrm{M}$ $=$ morning shift, $\mathrm{D}=$ day shift, $\mathrm{N}=$ night shift, $\mathrm{I}=$ the dividing line between two days, $+=$ no night between the shifts.

Sleepiness in shift combinations ending with the night shift

The prevalence of severe sleepiness in the night shift varied between $36 \%$ and $62 \%$ among the drivers and between $25 \%$ and $58 \%$ among the controllers in the various shift combinations (Fig. 1). The overall prevalence of severe sleepiness was $50.5 \%$. In the beginning of the night shift, severe sleepiness was present only rarely, but markedly more often during and at the end of the shift.

The generalised linear model for repeated measurements showed that work or sleep related explanatory variables were not significantly associated with the prevalence of severe sleepiness (Table 1). Of the individual factors, only subjective sleep need was associated significantly $\left(\chi^{2}(1)=12.74\right.$, $\mathrm{p}<.001)$ with severe sleepiness. One hour increase in sleep need increased the odds for the occurrence of severe sleepiness by $65 \%$. The amount of experienced sleep need varied between 4 and $12 \mathrm{~h}$ (median $8 \mathrm{~h}$ ). Also having at least one child less than $7 \mathrm{yr}$ of age tended to increase the risk for sleepiness in the night shift, even though the overall association between having at least one child and the occurrence of severe sleepiness at work was not significant $\left(\chi^{2}(2)=5.18, p=.075\right)$. None of the explanatory variables 


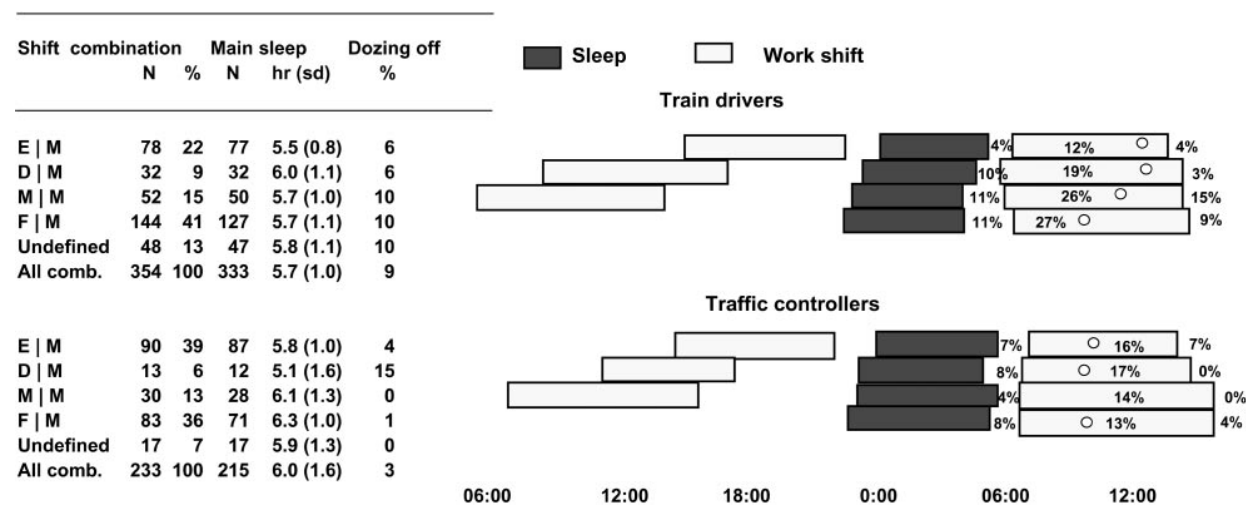

Fig. 2. The prevalence of severe sleepiness (KSS 7 or higher) of the train drivers and traffic controllers in the beginning, at any time (in the middle of the bar) and at the end of the morning shift in various shift combinations.

The mean timing of sleep and work periods in the shift combinations are also presented. White dots inside the morning shifts denote the mean timing of dozing off at work.

Table 2. A generalised linear model for repeated measurements for the occurrence of severe sleepiness during the morning shift

\begin{tabular}{|c|c|c|c|c|c|}
\hline Explaining variable & Categories / units & Odds ratio & $95 \%$ confidence & limits & $\mathrm{p}$ value \\
\hline \multirow[t]{2}{*}{ Age } & $>=45 \mathrm{yr}$ & .46 & .20 & 1.1 & .07 \\
\hline & $<45$ yr (reference) & 1 & - & - & \\
\hline \multirow[t]{2}{*}{ Occupation } & Driver & 1.02 & .47 & 2.20 & .96 \\
\hline & Controller (reference) & 1 & - & - & \\
\hline \multirow[t]{3}{*}{ Children at home } & $<7 \mathrm{yr}$ & 0.75 & 0.33 & 1.73 & .50 \\
\hline & $7-17 \mathrm{yr}$ & .47 & 0.22 & 1.00 & .051 \\
\hline & No children (reference) & 1 & - & - & \\
\hline Sleep need & Hour & 1.35 & 1.00 & 1.82 & .047 \\
\hline Main sleep period & Hour & 0.65 & 0.49 & 0.87 & .004 \\
\hline \multirow[t]{5}{*}{ Shift combination } & Undefined & 0.77 & 0.35 & 1.68 & .51 \\
\hline & $\mathrm{E} \mid \mathrm{M}$ & 0.94 & 0.48 & 1.80 & .84 \\
\hline & $\mathrm{D} \mid \mathrm{M}$ & 1.21 & 0.51 & 2.88 & .66 \\
\hline & $M \mid M$ & 1.01 & 0.48 & 2.14 & .97 \\
\hline & $\mathrm{F} \mid \mathrm{M}$ & 1 & - & - & \\
\hline Shift starting time & Time in hours & .82 & 0.57 & 1.17 & .28 \\
\hline Shift length & Time in hours & 1.13 & 1.01 & 1.26 & .03 \\
\hline
\end{tabular}

All explanatory variables are included in the model when the effect of one explanatory variable on the occurrence of severe sleepiness at work is tested. A total of 519 observations have been included in the analysis. Driver $=$ train driver, controller $=$ traffic controller, $\mathrm{M}$ $=$ morning shift, $\mathrm{D}=$ day shift, $\mathrm{E}=$ evening shift, $\mathrm{N}=$ night shift, $\mathrm{F}=$ free, $\mathrm{I}=$ the dividing line between two days.

interacted significantly with the shift combination factor.

When the sleep-related explanatory variables were removed from the statistical model the association between the shift combinations and sleepiness at work remained nonsignificant $(\mathrm{p}=0.56)$, but the effect of the shift length approached statistical significance $\left(\chi^{2}(1)=3.69, \mathrm{p}=.055\right)$. On average, each hour at work elevated the odds for severe sleepiness by 9\% (OR: 1.09 for each hour at work, $95 \%$ confidence interval 0.99-1.20).
Sleepiness in shift combinations ending with the morning shift

The prevalence of severe sleepiness in the morning shift varied between $12 \%$ and $27 \%$ among the drivers and between $13 \%$ and $17 \%$ among the controllers in the various shift combinations (Fig. 2). The overall prevalence of severe sleepiness in the morning shifts was $18.6 \%$.

The generalised linear model for repeated measurements revealed that of the sleep related explanatory variables, the length of the main sleep period was significantly associated $\left(\chi^{2}(1)=7.08, \mathrm{p}<.01\right)$ with severe sleepiness (Table 2$)$. On 
average, each hour of sleep decreased the odds for the occurrence of severe sleepiness by $35 \%$. Of the individual factors, the main effect of subjective sleep need approached the level of significance $\left(\chi^{2}(1)=3.82, p=.0506\right)$. On average, each hour of subjective sleep need increased the odds for the occurrence severe sleepiness by $35 \%$. Having at least one child between 7-17 yr of age tended to decrease the risk for sleepiness in the morning shift, but the overall association between having at least one child and the occurrence of severe sleepiness at work was not significant $\left(\chi^{2}(2)=3.81, \mathrm{p}=.15\right)$. Also the length of the morning shift affected sleepiness $\left(\chi^{2}(1)=3.96, \mathrm{p}<.05\right)$. Each hour at work increased the odds for the occurrence of severe sleepiness by $13 \%$. The shift combinations or the starting times of the shifts time were not significantly associated with the occurrence of severe sleepiness in the morning shift. None of the two-way interactions between the shift combination factor and one of the other explanatory factors was significant.

The exclusion of the sleep-related explanatory variables from the statistical model did not make the association between the shift combinations and sleepiness at work significant $(\mathrm{p}=.93)$. However, the association between the starting time of the morning shift and severe sleepiness at work became significant $\left(\chi^{2}(1)=8.42, \mathrm{p}<.01\right)$. On average, one hour delay in the starting time of the morning shift decreased the odds for the occurrence of sleepiness at work by $37 \%$ (OR: 0.63 for each hour of delay, $95 \%$ confidence interval 0.47-0.84).

\section{Discussion}

The main and new result of the present study was that there was no direct association between the various shift combinations and the occurrence of severe sleepiness in the night and morning shift. Other interesting findings were that the length of the night and morning shift and the starting time of the morning shift as well as some individual factors, such as subjective sleep need, were associated with the occurrence of severe sleepiness at work.

\section{Sleepiness in the shift combinations ending with the night shift}

The finding that the occurrence of severe sleepiness in the night shift was not associated with the various shift combinations, even when the length of prior sleep was not controlled for, is somewhat confusing. Our previous study showed that the length of the main sleep period was significantly shortened in certain shift combinations, especially when an early shift immediately preceded the night shift ${ }^{10)}$. On the other hand, our previous study also showed that a long period of free time before the night shift was not associated with a decrease in the prevalence of severe sleepiness ${ }^{9}$. In connection with the night shift, a free time period of $16 \mathrm{~h}$ or more before the shift was even associated with a higher risk for sleepiness at work than a free time period of 8-16 h. Härmä et al.$^{9)}$ suggested that train drivers and traffic controllers adjust their sleeping habits for each time-off period and shift combination. This pattern was clearly observable in our recent study, in which the percentage of afternoon napping was much higher in the morning+night and day+night shift combinations $(91 \%-71 \%)$ than in the free+night combination (59\%-33\%) among both occupational groups ${ }^{10}$.

It can be hypothesised that the non-significant association between the shift combination and severe sleepiness was due to the fact that the workers increased daytime napping before those night shifts that were preceded by an early shift. In an experimental study, daytime naps prior to the night shift have been shown to improve alertness during the shift ${ }^{15)}$. However, the association between the daytime naps and severe sleepiness was not significant in the present study. The same was true for the interaction between the shift combinations and daytime napping, meaning the alerting effect of the naps was not different between the various shift combinations.

One possible explanation for our findings is that at night the fatiguing effect of the circadian factor on alertness is so strong that up to $2.5 \mathrm{~h}$ reduction in the preceding main sleep period does not significantly increase the occurrence of severe sleepiness. For example, the study of Härmä et al. ${ }^{16)}$ showed that physiological sleepiness measured with the Maintenance Test of Wakefulness (MWT) was at the same level at night after an $8 \mathrm{~h}$ sleep on the preceding night as during a day that was preceded with only $2 \mathrm{~h}$ of nocturnal sleep.

Our measure of severe sleepiness was quite rough (at least one KSS rating of 7 or greater between the beginning and the end of the shift), which made it difficult to find differences among the various shift combinations. On the other hand, our previous study, in which the same measure of severe sleepiness was used as in the present study, showed that the occurrence of severe sleepiness varied greatly for example between the day and night shifts ${ }^{9)}$. Thus, it can be said that the differences in the occurrence of severe sleepiness are clearly slighter between various night shifts with a different shift history than between different shift types (e.g., night shift vs. day shift). Another methodological limitation concerns the way the KSS-ratings were collected. The subjects were asked to give their ratings in the beginning 
and at the end of each shift irrespective of the level of sleepiness and at any time between these time points when they felt sleepy. It is possible that at least some of the workers failed to report on sleepiness between the beginning and the end of the shift as they did not have to evaluate sleepiness systematically, e.g., in every two hours. These methodological limitations also hold for the results on the occurrence of sleepiness in the various shift combinations ending with the morning shift.

There may also be some intervening variables that can partly explain the non-significant relationship between the shift combinations and the occurrence of severe sleepiness. In this study, we could not control for the content of work, which has been shown to affect sleepiness to a great extent ${ }^{17)}$. It can be hypothesised that for example having two persons in the cabin or driving local traffic would keep the driver more vigilant than driving alone or having long distances between the stations. In addition, we did not control for other factors, for example meals or sleep disorders, which potentially could modify sleepiness. The small number of subjects and observations in certain shift combinations can be considered to increase the impact of these intervening factors on the results.

The length of the night shift tended to be positively associated with the occurrence of severe sleepiness, when the length of prior sleep was not controlled for. Also our previous study showed that each hour of the shift increased the risk for severe sleepiness by $15 \%$ when the data of the morning, day, evening, and night shifts were combined ${ }^{9)}$. This suggests that one effective way to improve alertness in the night shift is to keep the length of the shift relatively short.

\section{Sleepiness in the shift combinations ending with the} morning shift

The prevalence of severe sleepiness was 31\%-units lower for the morning shift than for the night shift, a finding that underlines the significance of the time of day for sleepiness. The non-significant association between the various shift combinations and severe sleepiness in the morning shift was probably due to the relatively small difference (about 30 $\min$ ) in the mean duration of the preceding sleep period between the combinations.

Similar to the night shift, the length of the morning shift was positively associated with the occurrence of sever sleepiness. One interesting question is whether this pattern was only due to shift length per se. Almost all short $(7.5 \mathrm{~h}$ or less) morning shifts ended at 2 p.m. or earlier. This means that only during the long shifts, the subjects had to work during the mid-afternoon hours (from 1 p.m. to 4 p.m.) to a great extent. In our recent study, we showed that EEG/EOGdefiend physiological sleepiness at work peaks just during these mid-afternoon hours ${ }^{17}$. Thus it remains open to what degree the positive association between the long working hours and the occurrence of severe sleepiness in the morning shift was due to time of the day on the one hand and to long working hours on the other hand.

The starting time of the morning shift was associated with the occurrence of severe sleepiness, but only when sleeping prior to the shift was not controlled for. This suggests that advancing of the shift starting time increased the occurrence of sleepiness by shortening the preceding sleep period. This result is somewhat contradictory with the study of Kecklund et al. ${ }^{18)}$, which showed no clear effect of earlier starting time of the morning shift on sleepiness at work. One likely explanation for this difference is that in the present study, the starting time of the morning shift varied between 0310 and 0655 a.m., whereas in Kecklund's study the earlier shift started at 0630 a.m. and the later shift at 0830 a.m. Thus, the delay of the shift starting time seems to be a more effective means to decrease sleepiness in the early morning shifts than is the extension of the free time prior to the shift. This observation is probably due to the fact that advancing of bed time is difficult because of "the forbidden zone" for sleep in the early evening ${ }^{19)}$ and due to social reasons.

The association between sleepiness and individual factors

Experienced sleep need was the most significant individual factor explaining the occurrence of severe sleepiness. Each additional hour of sleep need increased the risk for the occurrence of severe sleepiness by $65 \%$ and $35 \%$ in the night and morning shifts, respectively.

One problem with the use of experienced sleep need as an explanatory variable for sleepiness is that the workers possibly estimated their sleep need on the basis of how sleepy they felt at work. In the present study, the questionnaire containing the question about sleep need was, however, given prior to the use of the sleep/work time diary. This different timing of the measurements probably decreased the probability for a circular argument. There are also two issues that suggest that the participants did not evaluate their sleep need only based on their experienced sleepiness in the night and morning shifts. In our previous study, we found that the participants with high sleep need also slept somewhat more in connection with the night and morning shifts than did the rest of the participants ${ }^{10)}$. This demonstrates that higher sleep need is not only associated with more frequently experienced sleepiness, but also with longer sleeping time. Secondly and perhaps even more importantly, we did not 
ask experienced sleep need specifically in relation to the morning and night shifts, but more generally irrespective of shifts or work. Thus, it is quite unlikely that the participants, who also worked evening and day shifts and had days off, would have evaluated their sleep need high just because of experienced sleepiness during the night or morning shifts. All in all, it seems that experienced sleep need is a critical factor in explaining the occurrence of severe sleepiness in the night and morning shifts.

Also having at least one child less than $7 \mathrm{yr}$ of age tended to increase the occurrence of severe sleepiness in the night shift. One factor behind this association may be that having a small child decreases the main sleep period and afternoon napping prior to the night shift, as found in our previous study ${ }^{10)}$.

We did not find any significant age effect on the occurrence of severe sleepiness at work. This result is consistent with some previous studies on train drivers, ${ }^{7,21)}$. In our previous study, we, however, found that the risk for the occurrence of severe sleepiness at work decreased with ageing among the train drivers, when the data of the morning, day, evening and night shifts were combined ${ }^{9}$. For the traffic controllers, the corresponding age effect was not found. Taken together, it seems that ageing does not increase a risk for sleepiness at work among traffic controllers and train drivers. There is, however, at least one critical issue one has to keep in mind: older workers are probably more selected to shift work than younger workers. This "healthy worker effect" is inevitably present in cross-sectional designs.

In conclusion, the present study suggests that the occurrence of severe sleepiness during the night and morning shifts is not significantly related to the order of the preceding shifts. Important factors to minimise the occurrence of severe sleepiness at work seem to be the short duration of the night shift and the avoidance of the very early starting times of the morning shifts. The results also support the view that certain individual factors, such as long sleep need, are also associated with an increased risk for severe sleepiness in the morning and night shifts.

\section{References}

1) Åkerstedt $\mathrm{T}$ (1995) Work hours and sleepiness. Neurophysiol Clin 25, 367-375.

2) Kecklund G, Åkerstedt $T$ (1995) Effects of timing of shifts on sleepiness and sleep duration. J Sleep Res $\mathbf{4}$, $47-50$.

3) Dinges D (1995) An overview of sleepiness and accidents. J Sleep Res 4, 15-22.
4) Foret J, Lantin G (1972) The sleep of train drivers: an example of the effects of irregular work schedules on sleep. In: Aspects of Human Efficiency, Diurnal Rhythm and Sleep Loss. eds. by Colquhoun W.P, 273-82, Academic Press, London.

5) Åkerstedt T, Torsvall L, Fröberg JE (1983) A questionnaire study of sleep/wake disturbances and irregular work hours. Sleep Res 12, 358.

6) Hak A, Kampman R (1981) Working irregular hours: complaints and state of fitness of railway personnel. In: Night and shift work. Biological and social aspects. eds. by Reinberg A, Vieux N, 229-236, Pergamon Press, Oxford.

7) Ingre M, Söderström M, Kecklund G, Åkerstedt T, Kecklund L (2000) Train drivers work situation: working hours, sleep, stress and safety. Stress Research Report 292, Stockholm (Swedish with an English abstract).

8) Torsvall L, Åkerstedt $\mathrm{T}$ (1987) Sleepiness on the job: continuously measured EEG changes in train drivers. Electroenceph Clin Neurophysiol 66, 502-11.

9) Härmä, M, Sallinen M, Ranta R, Mutanen P, Muller K (2002) The effect of an irregular shift system on sleepiness at work in train drivers and railway traffic controllers. J Sleep Res 11, 141-51.

10) Sallinen M, Härmä, M, Mutanen $P$, Ranta R, Virkkala J, Muller K (2003) Sleep-wake rhythm in an irregular shift system. J Sleep Res 12, 103-12.

11) Åkerstedt T, Gillberg M (1990) Subjective and objective sleepiness in the active individual. Int J Neurosci 52, 29-37.

12) Gillberg M, Kecklund G, Åkerstedt $T$ (1994) Relations between performance and subjective ratings of sleepiness during a night awake. Sleep 17, 236-41.

13) Liang KY, Zeger SL (1986) Longitudinal Data Analysis using Generalized Linear Models, Biometrika 73, 1322.

14) Diggle PJ, Liang KY, Zeger SL (1994) Analysis of Longitudinal Data. Clarendon Press, Oxford.

15) Macchi MM, Boulos Z, Ranney T, Simmons L, Campbell SS (2002) Effects of an afternoon nap on nighttime alertness and performance in long-haul drivers. Accid Anal Prev 34, 825-34.

16) Härmä $M$, Suvanto $S$, Popkin $S$, Pulli K, Mulder M, Hirvonen K (1998) A dose-response study of total sleep time and the ability to maintain wakefulness. J Sleep Res 7, 167-74.

17) Sallinen M, Härmä M, Akila R, Holm A, Luukkonen R, Mikola H, Müller K, Virkkala J (2004) The effects 
of sleep debt and monotonous work on sleepiness and performance during a 12-h dayshift. J Sleep Res 13, 285-94.

18) Kecklund G, Akerstedt T, Lowden A (1997) Morning work: effects of early rising on sleep and alertness. Sleep 20, 215-23.

19) Lavie P (1986) Ultrashort sleep-waking schedule. III. Gates and 'forbidden zones' for sleep. Electroenceph and Clin Neurophysiol 63, 414-25.
20) Torsvall L, Åkerstedt T, Gillberg M (1981) Age, sleep and irregular work hours. A field study with electroencephalographic recording, catecholamin excretion and self-ratings. Scan J Work Environ Health 7, 196-203.

21) Hildebrandt G, Rohmert W, Rutenfranz J (1974) On the influence of age on the frequency of lack of performance by engine-drivers of the Federal German Railway. Int Arch Arbeitsmed 32, 33-41 (German). 